\title{
Genetic diversity of Plasmodium falciparum infections in mild and severe malaria of children from Kampala, Uganda
}

\author{
Mpungu Steven Kiwuwa • Ulf Ribacke • Kirsten Moll • \\ Justus Byarugaba • Klara Lundblom • Anna Färnert • \\ Kironde Fred • Mats Wahlgren
}

Received: 20 June 2012 / Accepted: 27 January 2013 /Published online: 14 February 2013

(C) The Author(s) 2013. This article is published with open access at Springerlink.com

\begin{abstract}
Diversity in parasite virulence is one of the factors that contribute to the clinical outcome of malaria infections. The association between the severity of Plasmodium falciparum malaria and the number of distinct parasite populations infecting the host (multiplicity of infection) or polymorphism within any of the specific antigen genes was investigated. The study included 164 children presenting with mild and severe malaria from central Uganda where malaria is meso-endemic. The polymorphic regions of the circumsporozoite protein ( $c s p$ ), merozoite surface proteins 1 and 2 ( $m s p 1$ and msp2), and glutamate-rich protein ( $g l u r p$ ) were genotyped by polymerase chain reaction methods and fragment analysis by gel electrophoresis. In a subset of samples fragment analysis was also performed by fluorescent PCR genotyping followed by capillary electrophoresis. The multiplicity of infection (MOI), determined as the highest number of alleles detected within any of the four genetic loci, was significantly higher in severe than in mild malaria cases (mean 3.7 and 3.0, respectively, $P=0.002$ ). No particular genotype or allelic family of $m s p 1$ or $m s p 2$ was associated with severity of malaria, and nor did the genotyping
\end{abstract}

M. S. Kiwuwa $(\triangle) \cdot$ J. Byarugaba

Department of Paediatrics, School of Medicine, Makerere University College of Health Sciences, Kampala, Uganda

e-mail: mkiwuwa@yahoo.com

K. Fred

Department of Biochemistry, School of Biomedical Sciences, Makerere University College of Health Sciences,

Kampala, Uganda

U. Ribacke $\cdot \mathrm{K}$. Moll $\cdot$ M. Wahlgren

Department of Microbiology, Tumor and Cell Biology (MTC),

Karolinska Institutet, Stockholm, Sweden

K. Lundblom • A. Färnert

Department of Medicine, Infectious Diseases Unit,

Karolinska Institutet, Stockholm, Sweden method reveal any significant difference in MOI when only assessed by $m s p 2$ genotyping. Severity of malaria was not linked to the predominance of any particular $m s p 1$ or $m s p 2$ allelic types, independent of methods used for genotyping. Monitoring the dynamics of multiple clone infections in relation to disease outcome, host immune status and genetic factors will provide more insight into parasite virulence mechanisms.

\section{Background}

Molecular epidemiological studies of malaria can be used to study the genetic diversity of infections in relation to various factors such as transmission intensity, disease phenotype and host immunity. Individuals infected by Plasmodium falciparum often consist of genetically distinct parasite populations, i.e. clones of the same parasite species (de Roode et al. 2005; Read and Taylor 2001). When clones compete for resources on exposure to host immune responses, their population dynamics can be affected (de Roode et al. 2005). Thus clone competition might affect host morbidity and transmission potential influencing the emergence of traits such as virulence and drug resistance. It has been postulated that reducing the number of genetically mixed infections may have health benefits through reduction of the level of within-host competition and hence the selection for reduced virulence (Galvani 2003). The clinical manifestations of malaria are quite diverse, ranging from asymptomatic parasitaemia, mild malaria to potentially fatal conditions such as severe anaemia, metabolic acidosis, coma and multi-organ failure (Miller et al. 2002). Although the molecular basis of severe malaria has been well studied in recent years, determinants of the clinical outcomes of malaria remain unknown (Conway 2007). Complex interactions of host, parasite and environmental factors are thought to contribute to the clinical outcome of malaria (Miller et al. 2002). One 
aspect of virulence in malaria refers to the harm done to the human host following an infection in terms of morbidity and mortality (Read 2007). The main virulence factors include the ability to induce binding of infected red blood cells (RBCs) to the vascular endothelium (cytoadherence) and to non-infected erythrocytes (rosetting) or to other infected erythrocytes (autoagglutination; Chen et al. 1998), and subsequent RBC microvasculature sequestration (Miller et al. 2002). The evolution from an uncomplicated to a severe infection such as cerebral malaria is not well understood. It is likely that the expression of specific binding phenotypes will result in distinct patterns of sequestration and pathogenic consequences (Mackintosh et al. 2004). Some investigators have reported more frequent binding to multiple receptors of isolates from children causing severe malaria vs mild malaria (Heddini et al. 2001). Consequently there is lack of clear understanding whether multiple adhesion of parasitized RBCs found in patients with severe malaria is due to the occurrence of several binding events arising from a single clonal population of parasites, or if the observation is due to several infecting clones exhibiting distinct receptor specificities. The repertoire of multiple infections may also induce the production of and/or the release of various pro-inflammatory cytokines that may be more difficult to control by the immune system, resulting in severe disease (John et al. 2008).

The relationship between the number of infecting clones in P. falciparum, multiplicity of infection (MOI) and severity of clinical episodes is not well understood and the findings have been inconsistent (Table 1). Some studies have demonstrated a higher MOI in severe cases (Ranjit et al. 2005; Rout et al. 2009) and in others similar numbers of genotypes per infection between the categories have been observed (A-Elbasit et al. 2007; Conway et al. 1991; Durand et al. 2008; Kun et al. 1998; Nielsen et al. 2002). Besides MOI, it is also not yet clear whether there are gene polymorphisms that cause some $P$. falciparum parasites to be inherently more virulent than others. Some studies have reported an association of particular $m s p 1$ or $m s p 2$ allelic types and severe malaria (Ariey et al. 2001; Kun et al. 1998), whereas others did not find such an association (Durand et al. 2008; Robert et al. 1996; Rout et al. 2009). Prior studies in Uganda have examined the relationship between MOI and the response to anti-malarial therapy or parasite densities in areas of differing endemicity (Cattamanchi et al. 2003; Kyabayinze et al. 2008; Peyerl-Hoffmann et al. 2001). However, no studies have been undertaken in Uganda to compare MOI in mild and severe malaria among children. The genetic diversity of $P$. falciparum parasites obtained from children presenting with severe or mild (uncomplicated malaria) from Kampala in Uganda was investigated. The aim of this study was to examine whether the severity of malaria episode was associated with multiplicity of infection, and/or a particular allelic family $m s p 1$ or $m s p 2$ genotype prior to initiation of anti-malarial treatment. Polymorphisms within the four antigen genes, namely the merozoite surface protein-1 (mspl), merozoite surface protein-2 (msp2), glutamate-rich protein (glurp) and circumsporozoite protein (csp) were analysed. The use of multiple markers may enhance the detection of diversity at

Table 1 Multiplicity of infection and severity of malaria

Mean multiplicity of infection (MOI)

Number of isolates

\begin{tabular}{|c|c|c|c|c|}
\hline Site (reference) & Genotypic marker & Uncomplicated malaria $^{\mathrm{a}}$ & Severe malaria ${ }^{\mathrm{b}}$ & $P$ value \\
\hline Gambia (Conway et al. 1991) & $m s p 1, m s p 2$ & $2.04(118)$ & $2.11(35)$ & $>0.05$ \\
\hline Senegal (Roberts et al. 1996) & msp1, msp2, glurp, csp, hrp1 & $3.1(30)$ & $2.2(56)$ & $<0.05$ \\
\hline Gabon (Kun et al. 1998) ${ }^{\mathrm{c}}$ & $m s p 1, m s p 2$ & NR (100) & NR (100) & $>0.05$ \\
\hline Ghana (Nielsen et al. 2002) & msp1, msp2, glurp & $2.1(28)$ & $2.0(34)$ & $>0.18$ \\
\hline India (Ranjit et al. 2005) ${ }^{\mathrm{d}}$ & msp1, msp2, glurp, hrp1 & NR (40) & NR (35) & $<0.001$ \\
\hline Gabon (Mayengue et al. 2007) & $m s p 1, m s p 2$ & $2.9(30)$ & $3.0(30)$ & $>0.05$ \\
\hline Sudan (A-Elbasit et al. 2007) & $m s p 2$ & $1.53(103)$ & $1.5(106)$ & $>0.05$ \\
\hline Nigeria (Amodu et al. 2008) & $m s p 2$ & $2.1(144)$ & $1.4(131)$ & $<0.05$ \\
\hline Madagascar (Durand et al. 2008) & $m s p 2$ & $3.72(134)$ & $3.73(102)$ & $>0.05$ \\
\hline India (Rout et al. 2009) & $m s p 1, m s p 2$ & $2.5(48)$ & $3.1(48)$ & 0.002 \\
\hline
\end{tabular}

$N R$ indicates not reported.

${ }^{a} \mathrm{UM}$, uncomplicated malaria is defined as acute falciparum malaria in the absence of danger signs or evidence of severe malaria

${ }^{\mathrm{b}} \mathrm{SM}$, severe malaria denotes cerebral malaria, severe malarial anaemia, respiratory distress, prostration, hypoglycaemia or other (World Health Organization 1990, 2000) criteria defining severe malaria.

${ }^{\mathrm{c}}$ Severe malaria cases comprised of severe malarial anaemia (haemoglobin $<5 \mathrm{~g} / \mathrm{dl}$ ) and hyperparasitaemic cases; mild malaria cases included for haemoglobin levels $>8 \mathrm{~g} / \mathrm{dl}$.

${ }^{\mathrm{d}}$ Severe malarial anaemia defined as haemoglobin $<7 \mathrm{gm} / \mathrm{dl}$; association of multiple allele frequency only significant for the $m s p 2$ allele. 
different polymorphic loci (Babiker et al. 1999). PCR genotyping methods were used to characterize parasite populations in which allelic variants can be simply distinguished by size following electrophoresis in agarose gels (Doolan 2000; Wooden et al. 1993). Furthermore, in a secondary analysis of a subset of samples, the conventional gel electrophoresis method was compared to fluorescent PCR coupled with highresolution capillary, electrophoresis (Liljander et al. 2009). Previous studies have revealed better resolution for detection of msp2 alleles by use of capillary electrophoresis (Falk et al. 2006; Liljander et al. 2009).

\section{Methods}

Study setting and participant recruitment

The study participants were recruited from the Paediatric Outpatient Assessment Centre and Acute Care Units of Mulago National Referral Hospital in Kampala and from Kasangati Health Centre IV in Wakiso District. Kampala District lies within Central Uganda. The district is bordered by Wakiso District to the south, the west and the north, and by Kira Municipality to the east. The coordinates of the district are: $0^{\circ} 19^{\prime} \mathrm{N} 32^{\circ} 35^{\prime} \mathrm{E}$ at an altitude of $1,200 \mathrm{~m}$. Malaria in this area is meso-endemic, with two peaks following the rainy seasons in March to May and September to November. Kampala features a tropical wet and dry climate. The average maximum daytime temperature in January is $28^{\circ} \mathrm{C}$, dropping to an average maximum of around $25^{\circ} \mathrm{C}$ in July. Mean annual precipitation is $1,174 \mathrm{~mm}$. Anopheles gambiae is the predominant mosquito vector. The estimated annual $P$. falciparum entomological inoculation rate (EIR) was found to be six infective bites per person per year in the neighbouring Jinja district (Okello et al. 2006). Mulago Hospital provides services to Kampala's population of about 1,720,000 (Uganda Bureau of Statistics, Statistical Abstract 2012) and surrounding districts plus upcountry referrals. During December 2007 to September 2008, children aged between 6 months and 6 years from the selected clinical settings were consecutively recruited to participate in the study according to the World Health Organization (WHO) criteria for the classification of malaria severity (World Health Organization 2000). The eligibility criteria for severe malaria cases included: (1) fever (axillary temperature $\geq 37.5^{\circ} \mathrm{C}$ ) or history of fever in the previous $24 \mathrm{~h}$, (2) $P$. falciparum infection with a parasite density of at least 2,000 asexual forms per microlitre of blood identified microscopically on thick blood smears, (3) no evidence of concomitant febrile illness and (4) the presence of one of the following clinical features: repeated convulsions $(>2$ in preceding $24 \mathrm{~h}$ ), altered consciousness, lethargy, prostration, inability to drink or breast feed, repeated vomiting, severe anaemia (haemoglobin level $<5.0 \mathrm{~g} / \mathrm{dl}$ ), respiratory distress (nasal flaring, in-drawing [recession] of the bony structure of the chest wall, or deep breathing) or jaundice. The inclusion criteria for mild (uncomplicated) malaria cases were parasite density $\geq 2,000$ parasites $/ \mu$ l, fever (axillary temperature $\geq 37.5^{\circ} \mathrm{C}$ ) or history of fever in the previous $24 \mathrm{~h}$, and absence of danger signs or evidence of severe malaria. Both severe and mild malaria patients were recruited simultaneously from each site. Patients with known chronic disease conditions, children receiving anti-retroviral therapy, or had an intake of anti-malarial drugs within the preceding 2 weeks were excluded. Written informed consent was obtained from parents or legal guardians of children who provided a blood sample for further studies prior to their enrolment. The Ethics Committee of the Faculty of Medicine, Makerere University and the Uganda National Council of Science and Technology approved the study.

\section{Clinical and laboratory investigations}

Upon enrolment, detailed clinical histories and examinations were undertaken from both groups of patients who were followed up until recovery. All participants received treatment according to the national treatment guidelines. A series of biochemical, haematological and other pathological investigations were performed at laboratories based in Kampala (LMK and Makerere University Biochemistry laboratories) and at Karolinska Institutet. Malaria was diagnosed by microscopy. Thick smears were prepared following the Lambaréné method (Kremsner et al. 1988). Each high-power field (HPF) of a thick smear was estimated to be approximately $1 / 630$ of a microlitre of blood (on a standard microscope at $\times 1,000$ magnification) and parasite counts were estimated for $20 \mathrm{HPF}$ or at least $100 \mathrm{HPFs}$ before declaring a smear negative. For internal quality control, $20 \%$ of the slides were read by an independent microscopist. A third reader resolved discrepant results. Clinical P. falciparum isolates (2-4 $\mathrm{ml}$ of venous blood) were collected by venipuncture into EDTA-coated tubes from patients before treatment was initiated (the procedure did not unduly delay the treatment).

\section{Isolation and purification of parasite DNA}

Whole blood treated with EDTA anti-coagulant was layered over a density gradient consisting of Polymorphprep ${ }^{\mathrm{TM}}$, (Axis-Shield) and centrifuged at $500 \times g$ for $35 \mathrm{~min}$ at room temperature. This method can concentrate parasitized RBCs effectively and recover a high level of malarial parasites ensuring minimal contamination with human host WBCs. Parasite DNA was then isolated from $200 \mu \mathrm{l}$ of parasitized packed cell volume (PCV) blood using the Easy-DNA ${ }^{\mathrm{TM}}$ Kit (Invitrogen), following the supplier's recommendations 
with minor modifications. In brief, parasite genomic DNA containing aqueous phase, once extracted with $25: 24: 1$ phenol/chloroform/isoamyl alcohol (Sigma), was treated with RNase before two additional rounds of extraction and subsequently the DNA concentration and quality were measured using a Nanodrop ${ }^{\mathrm{TM}}$ method (Thermo Fisher Scientific). Parasite DNA was re-suspended in $20 \mu \mathrm{l}$ of nuclease free water and stored at $-20{ }^{\circ} \mathrm{C}$ and later diluted to standardized concentrations during performance of PCR assays.

Genotyping of $P$. falciparum msp1, msp2, glurp and $c s p$ genes by gel electrophoresis

The polymorphic genetic loci of $m s p 1, m s p 2$, glurp and csp resulting from repetitive sequences where the number of a given repeat unit can vary between parasite lines, were used for genotyping of parasite populations in this study. Furthermore, for $m s p 1$ and $m s p 2$, the presence of unique sequences can be used to divide the variants into distinct allelic families. The polymorphic repetitive regions block 2 of $m s p 1$ and block 3 of $m s p 2$ were amplified by nested PCR, and RII repeat region of glurp by semi-nested PCR using the primers described by Doolan (2000) and Snounou et al. (1999). In the nest 1 reactions, primer pairs corresponding to conserved sequences spanning the polymorphic regions of each gene were included in separate reactions. The product generated in the first reaction was used as a template in six separate second nest 2 reactions, using in each case a specific primer pair in order to determine the presence of allelic variants from the K1, MAD20 and RO33 families of msp 1 block 2 , the 3D7/IC and FC27 families of $m s p 2$ repeats, and the RII blocks of glurp. The sequence of the primers used to amplify the central region of the csp gene were 5'-ATAGTAGATCAC TTGGAGA-3' (CSF) and 5'-GCATATTGTGACCTTG TCCA-3' (CSR) using a non nested regular PCR approach (Wooden et al. 1993). Each genotype was characterized by the size corresponding to its PCR products following agarose gel electrophoresis. The description of each isolate included the number of genotypes and the size of the corresponding PCR products within the isolate, as well as allelic types (i.e., families) of $m s p 1$ and $m s p 2$. Each polymorphic domain was amplified from $1 \mu \mathrm{l}$ of DNA solution $(\sim 10 \mathrm{ng} / \mu \mathrm{l})$ in a $25 \mu \mathrm{l}$ reaction mixture containing $250 \mathrm{nM}$ of each primer (except for glurp nest 1 primers which were used at a concentration of $125 \mathrm{nM}$ ), $2 \mathrm{mM} \mathrm{MgCl} 2,125 \mu \mathrm{M}$ of each dNTP, $0.5 \mathrm{U}$ Taq polymerase (Invitrogen) and PCR buffer.

The thermocycling conditions in thermocycler (Applied Biosystems) for $m s p 1, m s p 2$ and glurp nest 1 reactions were as follows: 5 min at $95{ }^{\circ} \mathrm{C}$, followed by 25 cycles for 2 min at $58^{\circ} \mathrm{C}, 2$ min at $72{ }^{\circ} \mathrm{C}$ and $1 \mathrm{~min}$ at $94^{\circ} \mathrm{C}$ then final annealing for $2 \mathrm{~min}$ at $58{ }^{\circ} \mathrm{C}$ and final extension of $5 \mathrm{~min}$ at $72{ }^{\circ} \mathrm{C}$. For the nest 2 PCR reactions, similar conditions were used for denaturing and extension, with the annealing temperature set at $61{ }^{\circ} \mathrm{C}$ for 30 amplification cycles. Similar PCR amplification reaction conditions were used for the $c s p$ locus with the annealing temperature set at $55^{\circ} \mathrm{C}$ for 35 cycles. The amplified PCR products were either stored at $+4{ }^{\circ} \mathrm{C}$ or analysed immediately by electrophoresis on a $2 \%$ molecular grade agarose gel (Biorad) and visualized by UV transilluminator after gel red (Biotium) staining. The sizes of the PCR products were estimated using Imagelab software version 3.0 (Biorad) with the sizes computed automatically by the software based on the 100 base pairs DNA ladder calibrator (Fermentas).

Fragment analysis by capillary electrophoresis

For the comparison of genotyping methods, a subset of DNA samples was randomly selected from the original sample and genotyped for $m s p 2$ by fluorescent-label PCR (Liljander et al. 2009). In brief, the PCR included the same initial amplification of the outer $m s p 2$ domain as described above, followed by two separate nested reactions with fluorescently labelled primers for the two allelic families of msp2 (FC27 and IC/3D7). Amplified products were analysed by capillary electrophoresis in a DNA sequencer (3730, Applied Biosystems) using GeneMapper software (Applied Biosystems).

\section{Definitions}

The detection of a single PCR fragment for each locus was classified as an infection with one parasite genotype (single genotype infection). The detection of more than one PCR fragment for either $m s p 1, m s p 2$, glurp or $c s p$ loci (i.e. an infection with more than one parasite genotype) was considered as multiple $P$. falciparum genotype infection. For a particular sample analysed, the number of (bands) observed for each of the genetic markers is determined (which for msp1 and msp 2 included adding the bands observed for the $\mathrm{K} 1$, MAD20 and RO33 families as well as the FC27 and 3D7/IC families respectively) Doolan (2000). MOI was defined as the number of parasite genotypes per infected patient, calculated by combining the $m s p 1, m s p 2$, glurp and csp PCR genotyping results. The maximum number of bands detected whatever the locus was considered as the MOI of that infection.

\section{Statistical analysis}

The mean MOI was compared between mild and severe cases using the non-parametric Mann-Whitney $U$ test with adjustment for ties. The proportions of alleles observed at each genetic locus within each group were compared using the Chi-square test statistic. Odds ratios and $95 \%$ confidence intervals were computed as measures of effect. $P$ values less 
than 0.05 were considered significant. All statistical analyses were performed using SPSS statistical software, version 18.0.

\section{Results}

Clinical characteristics of the malaria cases at presentation

The study population comprised 164 patients of whom 82 $(50 \%)$ had severe malaria and $82(50 \%)$ mild malaria according to the WHO classification criteria. The main clinical manifestations and characteristics of the patients are summarized in Table 2. There was a tendency of lower age in children with severe malaria compared to mild malaria (mean 28.7 and 33 months respectively, $P=0.227$ ). Geometric mean parasite density was higher in patients with severe malaria compared to that in mild cases $(32,064$ vs $12,110 / \mu 1, P=0.02)$. The distribution of $\mathrm{ABO}$ blood groups was similar in mild and severe cases. In the severe malaria category, 16 (19.5\%) presented with respiratory distress as the most common syndrome, 9 (12.9\%) had severe malaria anaemia and 12 $(15.6 \%)$ had features suggestive of cerebral malaria (determined as altered level of consciousness with asexual P. falciparum parasitaemia and no other evident aetiology of coma). Three patients with severe malaria died. None of the patients with mild malaria developed features of severe disease and all of them were clinically cured after treatment with artemether/lumefantrine. The patients with severe malaria were initially treated with intravenous quinine followed by administration of oral quinine.

Genotyping of different antigens

Blood samples were available for 164 of the patients (82 mild and 82 of severe malaria cases) for whom genotyping was performed. PCR amplification was successful in 97, 98.8, 97 and $87.2 \%$ of samples for $m s p 1, m s p 2$, glurp and csp respectively; and in $100 \%$ of samples when results for the four genes were combined.

Genetic diversity of infection

Of the 164 P. falciparum isolates genotyped by gel electrophoresis, $131(80.4 \%)$ comprised multiple genotypes. Among children with mild malaria 19 (23\%) were carrying single genotypes and $63(77 \%)$ had multiclonal infections (range 2-7). In the severe malaria group, 13 (15.8\%) patients had single and $69(84.2 \%)$ had multiple genotypes (range 2-8). Severe malaria cases were more likely to have $\geq 3$ genotypes per infection compared to mild malaria cases, OR 2.5 (95\% CI, 1.3-4.9, $P=0.009)$. When selecting the maximum number of alleles in the respective markers for each sample, the mean MOI was higher in severe malaria cases compared to that in mild malaria cases (3.7 vs 3.0 respectively, $P=0.002$ ). A high degree of genetic diversity was detected at the mspl and msp2 loci whereas less
Table 2 Comparison of baseline clinical features among children with mild and severe malaria, Kampala, Uganda
Values are number (\%) unless otherwise stated, unarousable coma indicates Blantyre coma scale $<3$.

Where total variable frequencies are $<100 \%$, data was unavailable for some study participants.

${ }^{a}$ Nasal flaring, in drawing (recession) of the bony structure of the chest wall, or deep breathing

${ }^{\mathrm{b}}$ Altered level of consciousness with asexual Plasmodium falciparum parasitaemia and no other evident etiology for coma.

\begin{tabular}{|c|c|c|c|}
\hline Characteristic & Severe malaria $(n=82)$ & Mild malaria $(n=82)$ & $P$ \\
\hline Age, mean (SD), mo & $28.7(21.7)$ & $33.0(23.5)$ & 0.227 \\
\hline Male gender & $50(61)$ & $52(63.4)$ & 0.747 \\
\hline Axillary temperature, mean (SD), ${ }^{\circ} \mathrm{C}$ & $38.6(1.1)$ & $37.8(1.1)$ & $<0.001$ \\
\hline Malaria rapid diagnostic test & $57(96.68)$ & $67(95.7)$ & 0.776 \\
\hline Geometric mean parasite density/ $\mu 1$ & 32,064 & 12,110 & 0.02 \\
\hline Hyperparasitaemia ( $>250,000 / \mu 1$ of blood) & $8(13.8)$ & $2(2.6)$ & 0.014 \\
\hline$>1$ Convulsions in last $24 \mathrm{~h}$ & $26(31.7)$ & $0(0)$ & $<0.001$ \\
\hline Respiratory distress $^{\mathrm{a}}$ & $16(19.5)$ & $0(0)$ & $<0.001$ \\
\hline Clinical jaundice & $14(17.1)$ & $0(0)$ & $<0.001$ \\
\hline Severe anaemia, $\mathrm{Hb}<5 \mathrm{~g} / \mathrm{dl}$ & $9(12.9)$ & $0(0)$ & 0.001 \\
\hline Unarousable coma & $1(1.2)$ & $0(0)$ & 0.316 \\
\hline Cerebral malaria $^{\mathrm{b}}$ & $12(15.6)$ & $0(0)$ & $<0.001$ \\
\hline Splenomegaly & $27(33.3)$ & $3(3.7)$ & $<0.001$ \\
\hline Circulatory collapse & $7(9.0)$ & $0(0)$ & 0.006 \\
\hline Total leucocyte count $\left(\times 10^{3}\right)$, mean (SD) & $10.8(5.1)$ & $9.7(4.2)$ & 0.181 \\
\hline Platelet count $\left(\times 10^{3}\right)$ mean $(\mathrm{SD})$ & $169.5(106.7)$ & $203.8(124.8)$ & 0.103 \\
\hline \multicolumn{4}{|l|}{ ABO blood groups } \\
\hline$(\mathrm{A}, \mathrm{B}, \mathrm{AB})$ & $38(52.1)$ & $40(50.0)$ & 0.800 \\
\hline$(\mathrm{O})$ & $35(47.9)$ & $40(50.0)$ & \\
\hline Blood transfusion & $11(13.4)$ & $0(0)$ & 0.001 \\
\hline
\end{tabular}


Table 3 Genotyping of $m s p 1$ and msp2, glurp and csp antigen genes among mild and severe malaria cases using gel electrophoresis ( $n=164)$

\begin{tabular}{|c|c|c|c|c|c|c|c|}
\hline \multirow[t]{2}{*}{ Genes } & \multicolumn{3}{|c|}{ Severe malaria $(n=82)$} & \multicolumn{3}{|c|}{ Mild malaria $(n=82)$} & \multirow[t]{2}{*}{$P$ value } \\
\hline & Positive $n^{\mathrm{a}}(\%)$ & Fragment size range bp & MOI & Positive $n^{\mathrm{a}}(\%)$ & Fragment size range bp & MOI & \\
\hline mspl & & & 3.2 & & & 2.5 & $0.005^{\mathrm{b}}$ \\
\hline K1 & $68(82.9)$ & $150-340$ & & $70(85.4)$ & $130-135$ & & $0.833^{\mathrm{c}}$ \\
\hline MAD20 & $41(50)$ & $180-350$ & & $41(50)$ & $100-310$ & & $1.000^{\mathrm{c}}$ \\
\hline RO33 & $40(48.8)$ & $140-270$ & & $31(37.8)$ & $130-300$ & & $0.157^{\mathrm{c}}$ \\
\hline$m s p 2$ & & & 2.7 & & & 2.3 & $0.050^{\mathrm{b}}$ \\
\hline $\mathrm{FC} 27$ & $71(86.8)$ & $220-590$ & & $64(78.0)$ & $230-580$ & & $0.166^{\mathrm{c}}$ \\
\hline $\mathrm{IC}$ & $76(92.7)$ & $300-750$ & & $76(92.7)$ & $300-750$ & & $1.000^{\mathrm{c}}$ \\
\hline glurp & $80(97.6)$ & $360-1300$ & 1.7 & $79(86.3)$ & $360-1250$ & 1.6 & $0.297^{\mathrm{b}}$ \\
\hline $\operatorname{csp}$ & $74(90.2)$ & $540-1200$ & 1.19 & $69(84.1)$ & $550-1020$ & 1.07 & $0.041^{\mathrm{b}}$ \\
\hline Overall $\mathrm{MOI}^{\mathrm{d}}$ & & & 3.7 & & & 3.0 & $0.002^{\mathrm{b}}$ \\
\hline
\end{tabular}

\footnotetext{
${ }^{a}$ Number of children with detection of respective allele types, Mean MOI (multiplicity of infection).

${ }^{\mathrm{b}} P$ values from Mann-Whitney $U$ test

${ }^{\mathrm{c}}$ Chi-square tests

${ }^{\mathrm{d}}$ Determined by selecting the highest number of alleles detected in any of the four antigen genes.
}

diversity was observed at the glurp and csp loci (Table 3). The msp1 RO33 and msp2 FC27 allelic families were detected at higher frequencies in severe malaria compared to mild malaria cases, although these differences are not statistically significant. The distribution of the other family specific alleles among mild and severe malaria cases was not significantly different. The allele frequency within the different genetic markers is shown in Fig. 1. There was a trend towards a higher multiplicity of infection at younger age groups. Age did not however have an effect on the mean multiplicity of infection between mild and severe malaria cases, $P=0.487$ (Fig. 2). Considering the classification of severe malaria according to the three major syndromes, severe malarial anaemia combined with respiratory distress exhibited a higher MOI compared to cerebral malaria alone, (4.2 vs 3.4 respectively, $P=0.231$ ) based on data from gel electrophoresis. However, this syndromic classification approach was restricted to only $43 / 82$ of the severe malaria cases.

$m s p 2$ genotyping using gel $v s$ capillary electrophoresis-

A supplementary analysis was undertaken on a subset of 133 samples using fluorescent-label PCR $m s p 2$ genotyping followed by capillary electrophoresis. The analysis indicated a modest agreement on the observed number of alleles when compared with the conventional gel electrophoresis method (kappa=0.07). Irrespective of the method used, there was no significant difference in MOI between the mild and severe malaria cases with regard to $m s p 2$ diversity alone and nor did we detect a better resolution with the capillary method (Table 4).

\section{Discussion}

The main finding of this study is that the mean number of circulating $P$. falciparum genotypes in severe malaria cases was higher than that of isolates from the mild cases, when selecting the highest number of alleles detected in any of the four antigen genes ( $m s p 1, m s p 2, c s p$ and glurp). The diversity was higher within the $m s p 1$ and $m s p 2$ loci compared to glurp and $c s p$ loci presumably because the latter are less polymorphic. These findings seem to suggest that the use of single genetic markers may underestimate the diversity of infections. The observed results of a higher MOI are consistent with those of smaller studies done in India (Ranjit et al. 2004, 2005; Rout et al. 2009) but in contrast to reports from Senegal (Robert et al. 1996) or Nigeria (Amodu et al. 2008), where the severe malaria category had a lower MOI than the mild type; and also studies from Ghana (Nielsen et al. 2002), Gabon (Mayengue et al. 2007), Sudan (A-Elbasit et al. 2007) and Madagascar (Durand et al. 2008) where comparable levels of MOI were observed among mild and severe malaria cases. The studies in India included adult patients with severe malaria in whom severe complications developed following initial treatment at rural health centres (Ranjit et al. 2004, 2005; Rout et al. 2009). Robert et al. (1996) included adults and children as well as French military personnel among the locals. Similarly, the study population of Durand et al. (2008) comprised of both adults and children. The initial treatment among the study

Fig. 1 Frequency distribution of allele types among mild and severe clinical cases: a maximal number of alleles considering all four markers, b $m s p 1$ alleles, c msp 2 alleles, d glurp alleles and e $c s p$ alleles. Count indicates number of children in the respective groups 
a

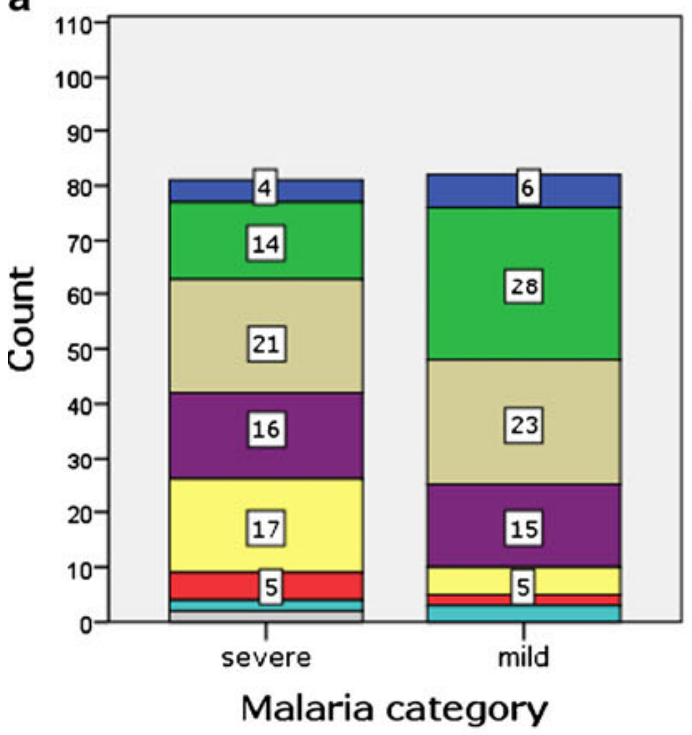

b

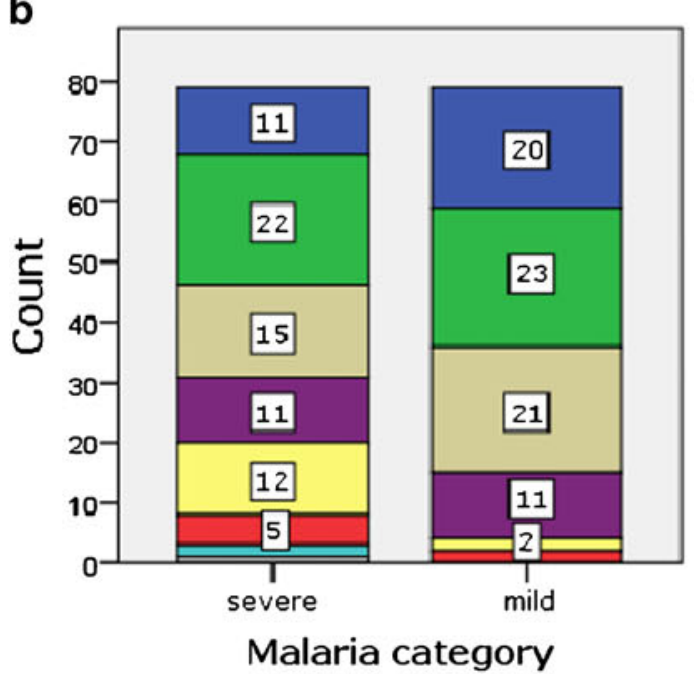

c

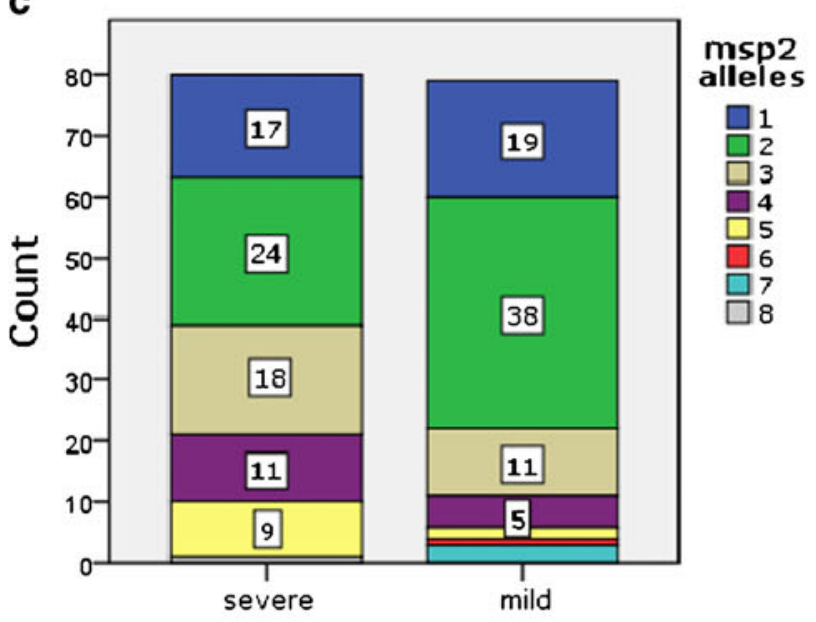

d

maximal allele frequency onsiderin markers

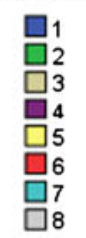

msp1 alleles

문

$\square 2$

\begin{tabular}{l}
4 \\
$\square$ \\
$\square$ \\
\hline
\end{tabular}

문

용

Malaria category

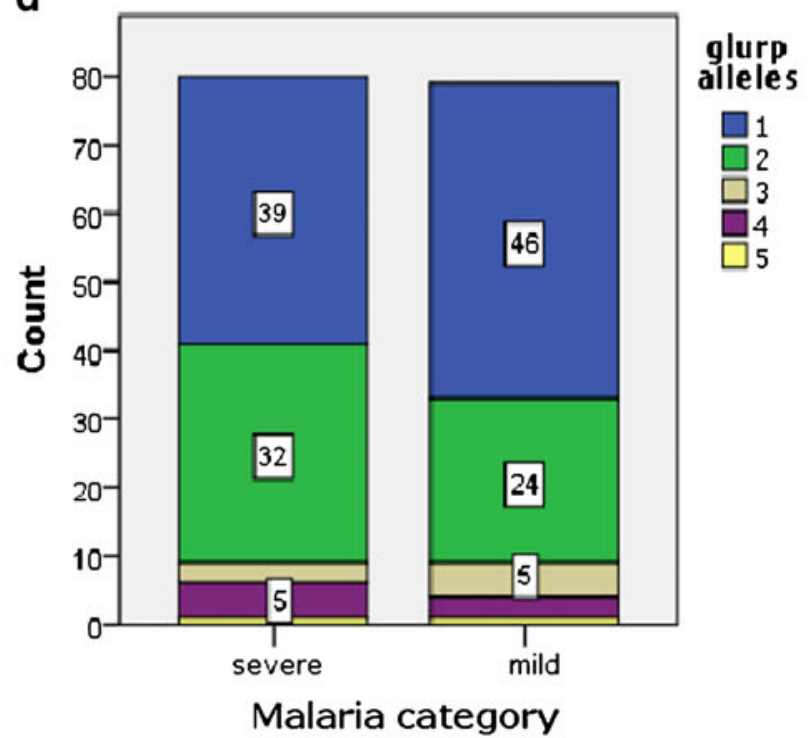

e

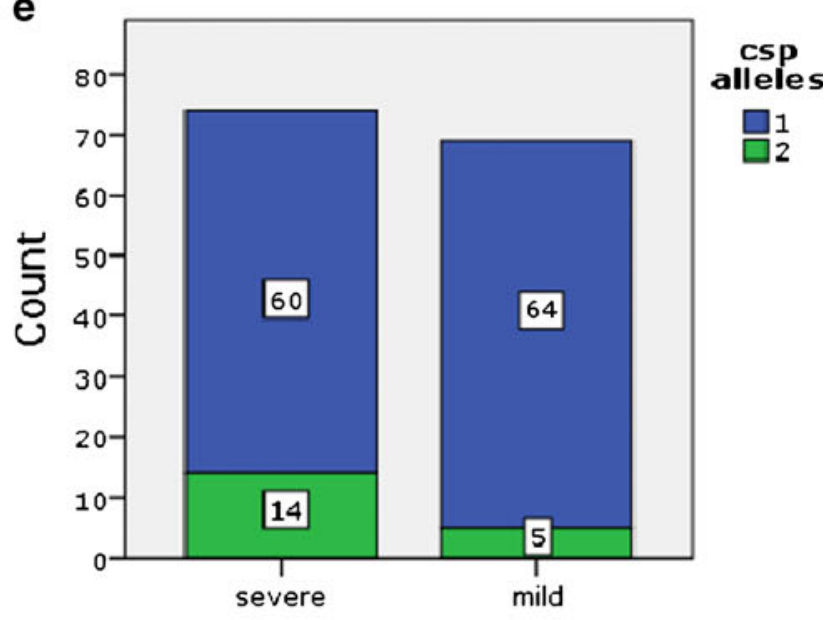

Malaria category 


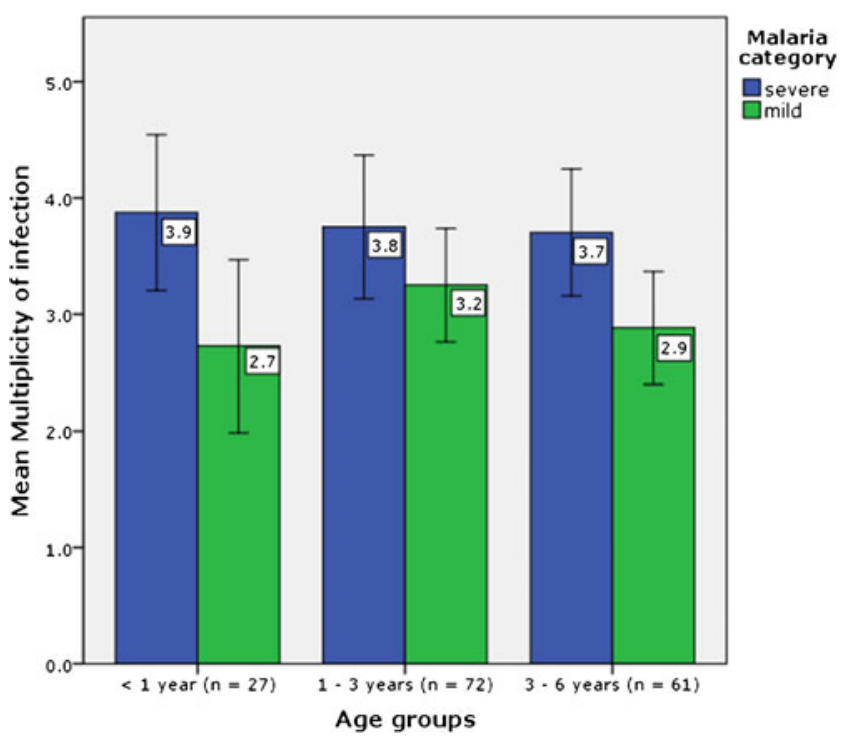

Fig. 2 Mean number of $P$. falciparum genotypes among children with mild and severe malaria according to age group. Error bars indicate $95 \%$ confidence intervals

cases from the different sites could have reduced the diversity of the isolates (Amodu et al. 2008; Durand et al. 2008; Ranjit et al. 2004, 2005; Robert et al. 1996; Rout et al. 2009). Additionally, differences in transmission intensity according to study sites may result in variable results when comparing MOI from different groups (A-Elbasit et al. 2007; Amodu et al. 2008; Robert et al. 1996). Differences in genotyping methods and/or interpretation of data as well as the heterogeneity of the various study populations may also partly explain the variability of results among studies. Due to the fluctuation of parasite density and complexity, a single peripheral blood sample may not reveal the full complexity of the parasite population harboured by an individual (Farnert 2008). Firstly, blood samples were collected once and secondly, PCR methods may fail to detect minority parasite strains that are not present in the peripheral blood at the time of sampling. The observation that children with non-cerebral malaria had higher MOI compared to those with cerebral malaria would require a larger study for confirmation (Montgomery et al. 2006). Children with severe malaria might have more sequestration (e.g. to capillaries in the brain) of certain parasite populations than mild malaria cases (Dembo et al. 2006; Montgomery et al. 2006). Alternatively MOI may also be regarded as an indicator of immune status in other vulnerable populations such as pregnant women. Pregnancy and low parity being associated with a higher MOI (Schleiermacher et al. 2001). In non-immune travellers, a higher MOI was similarly observed among severe malaria cases (Nicastri et al. 2008). Among children in Gabon, samples with the highest MOI significantly corresponded with lower antibody responses to specific asexual stage antigens, implying that low levels of acquired immunity may be related to the poor capacity to control infection (Mayeungue et al. 2009). Severe malaria may also be associated with higher parasite multiplication and rosette formation rates (Chotivanich et al. 2000; Le Scanf et al. 2008). However, in asymptomatic carriers in high endemicity areas, multiclonal infections have also been associated with a reduced risk of subsequent clinical malaria (Bereczky et al. 2007). For the secondary comparison of the gel electrophoresis method with high-resolution capillary electrophoresis (Liljander et al. 2009), no differences were observed with regard to MOI in severe or mild cases and neither was an improved resolution detected with the capillary method. Limitations in the sample size and use of a sole genetic marker may partly explain lack of a difference in the subgroup analysed. Further evaluation of a larger set of samples using additional polymorphic markers would assess allelic diversity more accurately using the capillary method (Mwingira et al. 2011).

The data of A-Elbasit et al. (2007), Durand et al. (2008) and Rout et al. (2009), in which no significant association was found between the occurrence of a specific genotype and the severity of malaria, support our findings. In French Guiana, it was demonstrated that a specific mspl allele bearing a specific variant gene (var-D) was associated with severe malaria (Ariey et al. 2001). However, this study was performed in a limited geographical area where parasite genetic diversity is reported to be low and thus, their results cannot be extrapolated to other areas. Ranjit et al. (2005) reported that MAD20/mspl and 3D7/msp2 alleles were over-represented in severe malaria cases in Orissa (India). Comparison of these findings with those of other groups demonstrates that the distribution of P. falciparum genotypes according to clinical severity seems to differ across geographic regions. A longitudinal study to further assess the extent of morbidity in relation to parasitic status, clonal diversity and host immune responses may provide more insight into the dynamics of virulence evolution in multiple parasite strain infections (Mayor et al. 2003).

Table 4 Comparison of msp2 genotyping multiplicity of infection among mild and severe malaria cases generated by two genotyping methods

\begin{tabular}{llll}
\hline Genotyping method & Severe malaria $(n=68)$ & Mild malaria $(n=65)$ & $P$ value \\
\hline PCR and gel electrophoresis & $2.51(0.97)$ & $2.74(0.96)$ & 0.183 \\
Fluorescent PCR and capillary electrophoresis & $2.43(1.42)$ & $2.25(1.24)$ & 0.435 \\
\hline
\end{tabular}

Values are mean (SD) unless otherwise indicated 


\section{Conclusions}

When selecting the highest number of alleles within the four analysed antigen genes, a higher MOI was detected in children with severe malaria. The differences in diversity between severe and mild cases were not as prominent within the individual markers and did not appear to be restricted to some particular genotypes or allelic family msp1 or msp2. Capillary electrophoresis offers the prospect to determine allelic diversity more accurately. Establishing the relationship between multiple clone infections, immune status, host genetic factors and the subsequent risk of clinical malaria will provide more insight into parasite virulence mechanisms.

Acknowledgments We would like to thank Judy Okiriza, Allan Lugajju and Moses Waswa for help with participant recruitment, sample preparations and microscopic analyses. LMK Laboratories performed haematological analyses. Anne Liljander provided technical assistance with fluorescent PCR and fragment analysis by capillary electrophoresis. We thank the participants and their guardians for agreeing to participate in the study and for the donation of clinical specimens. This work was supported by the Swedish Agency for International Development and the Belgian Technical cooperation. The funders had no role in the study design, data collection and analysis, decision to publish, or preparation of the manuscript.

Conflict of interest The authors declare that they have no competing interests.

Open Access This article is distributed under the terms of the Creative Commons Attribution License which permits any use, distribution, and reproduction in any medium, provided the original author(s) and the source are credited.

\section{References}

A-Elbasit IE, ElGhazali G, TM AE, Hamad AA, Babiker HA, Elbashir MI, Giha HA (2007) Allelic polymorphism of MSP2 gene in severe $P$. falciparum malaria in an area of low and seasonal transmission. Parasitol Res 102(1):29-34

Amodu OK, Oyedeji SI, Ntoumi F, Orimadegun AE, Gbadegesin RA, Olumese PE, Omotade OO (2008) Complexity of the msp2 locus and the severity of childhood malaria, in south-western Nigeria. Ann Trop Med Parasitol 102(2):95-102

Ariey F, Hommel D, Le Scanf C, Duchemin JB, Peneau C, Hulin A, Sarthou JL, Reynes JM, Fandeur T, Mercereau-Puijalon O (2001) Association of severe malaria with a specific Plasmodium falciparum genotype in French Guiana. J Infect Dis 184(2):237-241

Babiker HA, Ranford-Cartwright LC, Walliker D (1999) Genetic structure and dynamics of Plasmodium falciparum infections in the Kilombero region of Tanzania. Trans R Soc Trop Med Hyg 93 (Suppl 1):11-14

Bereczky S, Liljander A, Rooth I, Faraja L, Granath F, Montgomery SM, Farnert A (2007) Multiclonal asymptomatic Plasmodium falciparum infections predict a reduced risk of malaria disease in a Tanzanian population. Microbes Infect 9(1):103-110

Cattamanchi A, Kyabayinze D, Hubbard A, Rosenthal PJ, Dorsey G (2003) Distinguishing recrudescence from reinfection in a longitudinal antimalarial drug efficacy study: comparison of results based on genotyping of $m s p-1, m s p-2$, and glurp. AmJTrop Med Hyg 68(2):133-139

Chen Q, Barragan A, Fernandez V, Sundstrom A, Schlichtherle M, Sahlen A, Carlson J, Datta S, Wahlgren M (1998) Identification of Plasmodium falciparum erythrocyte membrane protein 1 (PfEMP1) as the rosetting ligand of the malaria parasite P. falciparum. J Exp Med 187:15-23

Chotivanich K, Udomsangpetch R, Simpson JA, Newton P, Pukrittayakamee S, Looareesuwan S, White NJ (2000) Parasite multiplication potential and the severity of Falciparum malaria. J Infect Dis 181:1206-1209

Conway DJ (2007) Molecular epidemiology of malaria. Clin Microbiol Rev 20(1):188-204

Conway DJ, Greenwood BM, McBride JS (1991) The epidemiology of multiple-clone Plasmodium falciparum infections in Gambian patients. Parasitology 103(Pt 1):1-6

de Roode JC, Helinski ME, Anwar MA, Read AF (2005) Virulence and competitive ability in genetically diverse malaria infections. Proc Natl Acad Sci U S A 102(21):7624-7628

Dembo EG, Phiri HT, Montgomery J, Molyneux ME, Rogerson SJ (2006) Are Plasmodium falciparum parasites present in peripheral blood genetically the same as those sequestered in the tissues? AmJTrop Med Hyg 74(5):730-732

Doolan DL (2000) Malaria methods and protocols. Humana Press, Inc., Totowa

Durand R, Ariey F, Cojean S, Fontanet A, Ranaivo L, Ranarivelo LA, Vonimpaisomihanta JA, Menard D, Pietra V, Le Bras J, Modiano D, Randrianarivelojosia M (2008) Analysis of circulating populations of Plasmodium falciparum in mild and severe malaria in two different epidemiological patterns in Madagascar. Trop Med Int Health 13(11):1392-1399

Falk N, Maire N, Sama W, Owusu-Agyei S, Smith T, Beck HP, Felger I (2006) Comparison of PCR-RFLP and Genescan-based genotyping for analyzing infection dynamics of Plasmodium falciparum. Am J Trop Med Hyg 74(6):944-950

Farnert A (2008) Plasmodium falciparum population dynamics: only snapshots in time? Trends Parasitol 24(8):340-344

Galvani AP (2003) Epidemiology meets evolutionary ecology. Trends Ecol Evol 18(3):132-139

Heddini A, Pettersson F, Kai O, Shafi J, Obiero J, Chen Q, Barragan A, Wahlgren M, Marsh K (2001) Fresh isolates from children with severe Plasmodium falciparum malaria bind to multiple receptors. Infect Immun 69(9):5849-5856

John CC, Park GS, Sam-Agudu N, Opoka RO, Boivin MJ (2008) Elevated serum levels of IL-1ra in children with Plasmodium falciparum malaria are associated with increased severity of disease. Cytokine 41:204-208

Kremsner PG, Zotter GM, Feldmeier H, Graninger W, Rocha RM, Wiedermann G (1988) A comparative trial of three regimens for treating uncomplicated falciparum malaria in Acre, Brazil. J Infect Dis $158(6): 1368-1371$

Kun JF, Schmidt-Ott RJ, Lehman LG, Lell B, Luckner D, Greve B, Matousek P, Kremsner PG (1998) Merozoite surface antigen 1 and 2 genotypes and rosetting of Plasmodium falciparum in severe and mild malaria in Lambarene, Gabon. Trans R Soc Trop Med Hyg 92(1):110-114

Kyabayinze DJ, Karamagi C, Kiggundu M, Kamya MR, WabwireMangen F, Kironde F, Talisuna A (2008) Multiplicity of Plasmodium falciparum infection predicts antimalarial treatment outcome in Ugandan children. Afr Health Sci 8(4):200-205

Le Scanf C, Vigan-Womas I, Contamin H, Guillotte M, Bischoff E, Mercereau-Puijalon O (2008) Rosetting is associated with increased Plasmodium falciparum in vivo multiplication rate in the Saimiri sciureus monkey. Microbes Infect 10:447-451

Liljander A, Wiklund L, Falk N, Kweku M, Martensson A, Felger I, Farnert A (2009) Optimization and validation of multi-coloured 
capillary electrophoresis for genotyping of Plasmodium falciparum merozoite surface proteins ( $m s p 1$ and 2). Malar J 8:78

Mackintosh CL, Beeson JG, Marsh K (2004) Clinical features and pathogenesis of severe malaria. Trends Parasitol 20:597-603

Mayengue PI, Kalmbach Y, Issifou S, Kremsner PG, Ntoumi F (2007) No variation in the prevalence of point mutations in the Pfert and Pfmdr1 genes in isolates from Gabonese patients with uncomplicated or severe Plasmodium falciparum malaria. Parasitol Res 100(3):487-493

Mayengue PI, Luty AJ, Rogier C, Baragatti M, Kremsner PG, Ntoumi F (2009) The multiplicity of Plasmodium falciparum infections is associated with acquired immunity to asexual blood stage antigens. Microbes Infect 11(1):108-14

Mayor A, Saute F, Aponte JJ, Almeda J, Gomez-Olive FX, Dgedge M, Alonso PL (2003) Plasmodium falciparum multiple infections in Mozambique, its relation to other malariological indices and to prospective risk of malaria morbidity. Trop Med Int Health 8:3-11

Miller LH, Baruch DI, Marsh K, Doumbo OK (2002) The pathogenic basis of malaria. Nature 415(6872):673-679

Montgomery J, Milner DA Jr, Tse MT, Njobvu A, Kayira K, Dzamalala CP, Taylor TE, Rogerson SJ, Craig AG, Molyneux ME (2006) Genetic analysis of circulating and sequestered populations of Plasmodium falciparum in fatal pediatric malaria. J Infect Dis 194(1):115-122

Mwingira F, Nkwengulila G, Schoepflin S, Sumari D, Beck HP, Snounou G, Felger I, Olliaro P, Mugittu K (2011) Plasmodium falciparum msp1, msp2 and glurp allele frequency and diversity in sub-Saharan Africa. Malar J 10:79

Nicastri E, Paglia MG, Severini C, Ghirga P, Bevilacqua N, Narciso P (2008) Plasmodium falciparum multiple infections, disease severity and host characteristics in malaria affected travellers returning from Africa. Travel Med Infect Dis 6(4):205-209

Nielsen MA, Staalsoe T, Kurtzhals JA, Goka BQ, Dodoo D, Alifrangis M, Theander TG, Akanmori BD, Hviid L (2002) Plasmodium falciparum variant surface antigen expression varies between isolates causing severe and nonsevere malaria and is modified by acquired immunity. J Immunol 168(7):3444-50

Okello PE, Van Bortel W, Byaruhanga AM, Correwyn A, Roelants P, Talisuna A, D'Alessandro U, Coosemans M (2006) Variation in malaria transmission intensity in seven sites throughout Uganda. AmJTrop Med Hyg 75:219-225

Peyerl-Hoffmann G, Jelinek T, Kilian A, Kabagambe G, Metzger WG, von Sonnenburg F (2001) Genetic diversity of Plasmodium falciparum and its relationship to parasite density in an area with different malaria endemicities in West Uganda. Trop Med Int Health 6(8):607-613

Ranjit MR, Das A, Chhotray GP, Das BP, Das BN, Acharya AS (2004) The PfCRT (K76T) point mutation favours clone multiplicity and disease severity in Plasmodium falciparum infection. Trop Med Int Health 9(8):857-861

Ranjit MR, Das A, Das BP, Das BN, Dash BP, Chhotray GP (2005) Distribution of Plasmodium falciparum genotypes in clinically mild and severe malaria cases in Orissa, India. Trans R Soc Trop Med Hyg 99(5):389-395

Read A (2007) Evolution of virulence: malaria, a "Case Study". In: Nesse R (ed) Evolution and Medicine: How New Applications Advance Research and Practice, The Biomedical \& Life Sciences Collection. Henry Stewart Talks Ltd, London

Read AF, Taylor LH (2001) The ecology of genetically diverse infections. Science 292(5519):1099-1102

Robert F, Ntoumi F, Angel G, Candito D, Rogier C, Fandeur T, Sarthou JL, Mercereau-Puijalon O (1996) Extensive genetic diversity of Plasmodium falciparum isolates collected from patients with severe malaria in Dakar, Senegal. Trans R Soc Trop Med Hyg 90(6):704-711

Rout R, Mohapatra BN, Kar SK, Ranjit M (2009) Genetic complexity and transmissibility of Plasmodium falciparum parasites causing severe malaria in central-east coast India. Trop Biomed 26(2):165-172

Schleiermacher D, Rogier C, Spiegel A, Tall A, Trape JF, MercereauPuijalon O (2001) Increased multiplicity of Plasmodium falciparum infections and skewed distribution of individual mspl and $m s p 2$ alleles during pregnancy in Ndiop, a Senegalese village with seasonal, mesoendemic malaria. AmJTrop Med Hyg 64(5-6):303-309

Snounou G, Zhu X, Siripoon N, Jarra W, Thaithong S, Brown KN, Viriyakosol S (1999) Biased distribution of $m s p 1$ and $m s p 2$ allelic variants in Plasmodium falciparum populations in Thailand. Trans R Soc Trop Med Hyg 93(4):369-374

Uganda Bureau of Statistics (UBOS) Statistical Abstract (2012) Government of Uganda, Ministry of Finance Planning and Economic Development. Kampala, Uganda

Wooden J, Kyes S, Sibley CH (1993) PCR and strain identification in Plasmodium falciparum. Parasitol Today 9(8):303-305

World Health Organization (1990) Severe and complicated malaria. Division of Control of Tropical Diseases. Trans R Soc Trop Med Hyg 84(Supplement 2):1-65

World Health Organization (2000) Severe falciparum malaria. Trans R Soc Trop Med Hyg 94(Supplement 1):1-90 